\title{
Structure and Expression Levels of Alleles of Citrus Zeaxanthin Epoxidase Genes
}

\author{
Aiko Sugiyama ${ }^{1 *}$, Yoshinori Ikoma ${ }^{2}$, Hiroshi Fujii ${ }^{2}$, Takehiko Shimada ${ }^{2}$, Tomoko Endo ${ }^{2}$, \\ Tokurou Shimizu² and Mitsuo Omura ${ }^{3}$ \\ ${ }^{1}$ The United Graduate School of Agricultural Science, Gifu University, Yanagido, Gifu 501-1193, Japan \\ ${ }^{2}$ National Institute of Fruit Tree Science (NIFTS), National Agriculture and Bio-oriented Research Organization (NARO), Okitsu- \\ nakacho, Shimizu, Shizuoka 424-0292, Japan \\ ${ }^{3}$ Faculty of Agriculture, Shizuoka University, Ohya, Suruga, Shizuoka 422-8529, Japan
}

The carotenoid metabolism enzyme zeaxanthin epoxidase (ZEP) is a key regulator of carotenoid accumulation in Citrus fruit. The expression level of the gene encoding this enzyme, $Z E P$, influences varietal differences in the conversion of zeaxanthin to violaxanthin during fruit development. To determine how this gene is regulated, we investigated the structure of its alleles and analyzed allele-specific $Z E P$ expression in heterozygous Citrus fruit. Four independent genomic sequences were isolated from a BAC library of Satsuma mandarin (Citrus unshiu Marc.). All $Z E P$ gene sequences in Satsuma mandarin consisted of 16 exons and 15 introns. Genetic analyses of hybrid populations and analyses of sequence variations identified that two of the sequences were alleles from the $Z E P-1 /-2$ locus, and others were from different locus/loci, $Z E P-3 /-4$, for which allelism has not been confirmed. The $Z E P-4$ allele was expressed in young-stage fruit, but not in mature-stage fruit, while $Z E P-1 /-2$ alleles were expressed as fruit matured. Our results showed that the newly identified isoform, $Z E P-4$, did not contribute to the accumulation of violaxanthin during fruit maturation. Expression levels of $Z E P-1 /-2$ alleles in the fruit of three heterozygous Citrus cultivars were compared using allele-specific RT-PCR. Transcripts of $Z E P$ - 1 alleles were more abundant than those of $Z E P$-2 alleles in all three cultivars. Sequence diversity among the 5' UTRs of $Z E P$ alleles was also analyzed. The implications of sequence diversity with respect to diversity of the expression and phylogenetic relationships among $Z E P$ genes in Citrus are discussed.

Key Words: alleles-specific transcript ratio, carotenoid, cis-element, fruit maturation, isoform.

\section{Introduction}

Altering the accumulation and composition of carotenoids in Citrus fruit is one of the major objectives of Citrus breeding programs. Carotenoids determine fruit color, and have roles as bio-functional compounds, for example, vitamin A and various compounds with anticancer activity (Nishino et al., 2000; Olson, 1989).

Carotenoid content and composition in Citrus fruit varies greatly among varieties, and depends on growing conditions (Gross, 1987; Ikoma et al., 2001; Kato et al., 2004). Satsuma mandarin (Citrus unshiu Marc.) and other mandarins accumulate $\beta$-cryptoxanthin predominantly in the flavedo and juice sacs in mature fruit (Goodner et al., 2001; Ikoma et al., 2001). In contrast, sweet orange (Citrus sinensis Osbeck) fruit accumulates violaxanthins, predominantly 9-cis-violaxanthin (Lee

Received; August 28, 2009. Accepted; March 18, 2010.

* Corresponding author (E-mail: osugi@affrc.go.jp). and Castle, 2001; Molnár and Szabolcs, 1980).

Understanding how carotenoid metabolism is regulated in fruit is key to increasing carotenoid content or altering its composition. Therefore, carotenoid biosynthesis and its regulation have been studied extensively in Citrus. Recently, expression profiles of genes encoding various carotenoid biosynthetic enzymes in Citrus fruit have been reported. Phytoene synthase (PSY) and $\beta$-ring hydroxylase $(\mathrm{HYb})$ are the major enzymes in the metabolic pathway. Transcript levels of PSY increased in the peel and juice sacs at the onset of rind coloration in Satsuma mandarin (Ikoma et al., 2001; Kim et al., 2001).

Kato et al. (2004) compared the expression level of genes affecting carotenoid content between Satsuma mandarin and 'Valencia' orange during fruit maturation. Their results showed that simultaneous increases in expression levels of PSY, phytoene desaturase (PDS), zeta-carotene desaturase $(Z D S)$, lycopene beta-cyclase $(L c y b)$, and zeaxanthin epoxidase $(Z E P)$ were correlated 
with the massive accumulation of $\beta, \beta$-xanthophyll $(\beta$ cryptoxanthin, zeaxanthin, and violaxanthin) in both the flavedo and juice sacs. In addition, accumulation of phytoene was initiated along with a decrease in the transcript level of $P D S$ in the flavedo of Satsuma mandarin. Together, these results suggested that types and levels of carotenoids are highly regulated by the expression of carotenoid biosynthetic genes during fruit maturation (Kato et al., 2004).

Carotenoid metabolism has been investigated at the gene expression level in many plant species aside from Citrus, such as Arabidopsis (Park et al., 2002; Pogson et al., 1996), tomato (Solanum lycopersicum; Fraser et al., 1994; Giuliano et al., 1993; Isaacson et al., 2002; Ronen et al., 1999), pepper (Capsicum annuum; Bouvier et al., 1996, 1998), and tobacco (Nicotiana tabacum; Busch et al., 2002); however, only a few studies have focused on the regulation of carotenoid metabolism genes. Welsch et al. (2003) reported that, in Arabidopsis, there are two main regions in the $P S Y$ promoter that regulate transcriptional activity. They also discovered a transcription factor gene, $R A P 2.2$, which interacts with the promoter motifs found in both $P S Y$ and PDS (Welsch et al., 2008). These analyses suggested that diversity among cis-elements as well as transcription factor genes would contribute to the genotype-dependent expression of genes involved in carotenogenesis.

Whereas some plants are genetically homozygous, such as Arabidopsis and rice, Citrus cultivars are usually highly heterozygous at many EST loci (Omura et al., 2000); therefore, the transcript levels in fruit represent the total expression from all alleles. For example, allelespecific expression profiles of the UDP-glucose: limonoid glucosyltransferase gene have been determined during fruit development in heterozygous Satsuma mandarin (Kita et al., 2003).

Here, to understand the role of cis-elements in expression, we investigated the allele-specific expression of ZEP in Citrus cultivars. ZEP catalyzes the conversion of zeaxanthin to violaxanthin in the carotenoid pathway, and possibly influences the accumulation of violaxanthin, which accumulates at high levels in fruit of $C$. sinensis but low levels in C.unshiu and 'Kiyomi' tangor (C. unshiu $\times$ C. sinensis).

Differences in the genomic structure of alleles associated with the expression level could be utilized to generate functionally defined DNA markers, which could be employed in Citrus breeding programs to alter carotenoid content. We investigated the genomic structure of ZEP genes, and examined diversity among alleles and its effects on gene expression in Citrus. To confirm the genetic background of allelism in ZEP genes, we analyzed gene sequences and expressions in three Citrus cultivars; 'Miyagawa wase' (C. unshiu) and 'Trovita' orange (C. sinensis), which differ in their carotenoid accumulation profile and in the expression profiles of carotenoid metabolism genes, and their hybrid
'Kiyomi' tangor accumulates violaxanthin at intermediate levels of the parents.

\section{Materials and Methods}

\section{Plant materials}

'Miyagawa wase' Satsuma mandarin (C. unshiu Marc.), 'Trovita' orange (C. sinensis Osbeck), and 'Kiyomi' tangor (C. unshiu $\times$ C. sinensis) were used for genomic sequence and expression analyses. The cultivars shown in Figure 1B were also used to analyze the polymorphism of the ZEP gene among cultivars. All cultivars used in this study were cultivated at the National Institute of Fruit Tree Science (NIFTS), Okitsu (Shizuoka, Japan). For segregation analyses, we also used hybrids between 'Kiyomi' tangor $\times$ 'Miyagawa wase', 'Jutaro unshiu' (C. unshiu Marc.) $\times$ 'Trovita' orange, 'Kiyomi' tangor $\times$ 'Clementine' (C. clementina hort. ex Tanaka), and 'Hirakisyu' (C. kinokuni hort. ex Tanaka) $\times$ 'Miyagawa wase'. Genomic DNA was isolated from leaves of the cultivars and hybrids using a Nucleon Phytopure extraction kit (GE Healthcare UK Ltd., Little Chalfont, Buckinghamshire, England). For expression analyses, in harvested fruits juice sacs were separated from rind, and juice sacs were immediately frozen in liquid nitrogen and kept at $-80^{\circ} \mathrm{C}$ until RNA extraction.

\section{Isolation and sequence analysis of ZEP}

The cDNA sequence of the ZEP gene in Citrus (DDBJ accession number $\mathrm{AB} 075547)$ was used to design the following primer pair: Forward, 5'-TTTATTTGGGCCA CAGGAAGC; Reverse, 5'-TCCCCAAGCAAAGTAAC ACGA. These primers were used to genotype and screen the BAC library derived from 'Miyagawa wase' (C. unshiu) (Shimada et al., 2005) by PCR, with DNAs isolated from BAC clones using a QIAGEN LargeConstruct Kit (Qiagen, Hilden, Germany). An insert from BAC screening was sequenced by primer walking, and primer sets designed from this sequence (Table 1 ) were used for primer walking of genomic DNAs of Citrus cultivars.

For genomic DNA and BAC DNA, PCR amplification was performed using AmpliTaq Gold DNA polymerase (Applied Biosystems, Foster City, CA, USA) protocols at an annealing temperature of $56^{\circ} \mathrm{C}$ for 35 cycles. Amplified PCR products were cloned into a pGEM $^{\circledR}$-T Easy vector (Promega, WI, USA), transformed into Escherichia coli strain XL-1 Blue, and sequenced using a BigDye Terminator v3.1 Cycle Sequencing Kit (Applied Biosystems) with an ABI PRISM 3100 Genetic Analyzer (Applied Biosystems).

Genetyx-Win ver. 8 (Software Development Co., Tokyo, Japan) and ATGC ver. 3.0.3 (Software Development Co.) were used for alignment analysis. A phylogenic tree was constructed using MEGA4 Build\#: 4026 (http://evolgen.biol.metro-u.ac.jp/MEGA/) by the $\mathrm{NJ}$ method. 

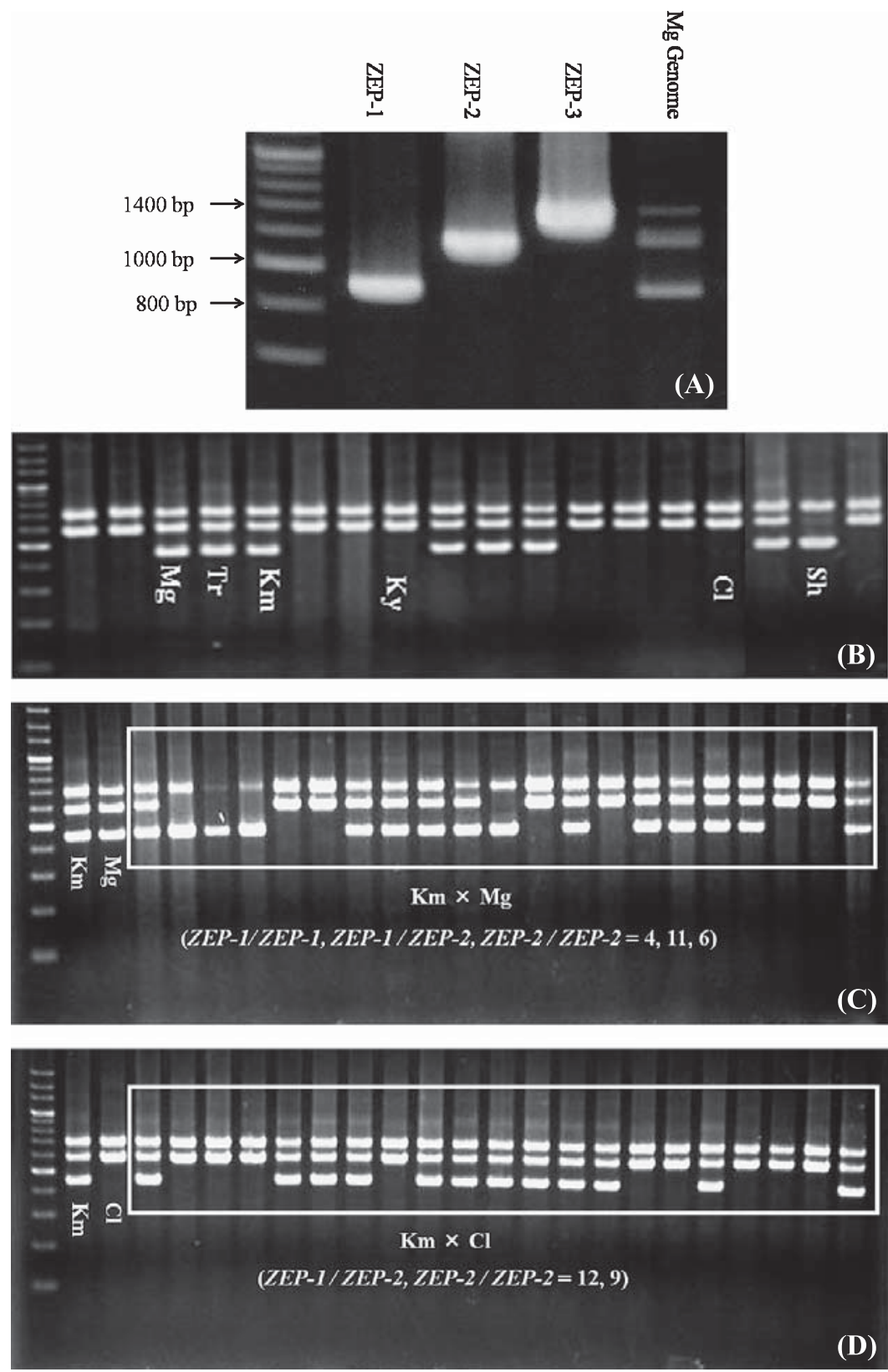

Fig. 1. PCR amplification patterns of Citrus cultivars and hybrid populations using ZEP primers. (A) Amplification pattern of each $Z E P$. These three ZEP PCR fragments were derived from each 'Miyagawa wase' BAC clone. The amplicon sizes of ZEP-1, ZEP-2 and ZEP-3 are $887 \mathrm{bp}, 1166 \mathrm{bp}$ and $1396 \mathrm{bp}$, respectively. PCR fragment in right lane was derived from 'Miyagawa wase' genome. (B) Citrus cultivars, from left to right: Kumquat, Citron, Satsuma mandarin ('Miyagawa wase', Mg), 'Trovita' orange (Tr), 'Kiyomi' tangor (Km), Tachinbana, Koji, 'Hirakisyu' (Ky), 'Mato' pumello, Kunenbo, 'King' mandarin, 'Ohta' ponkan, 'Dancy' tangerin, Mediterrranean mandarin, 'Clementine' (Cl), 'Seminor', 'Seiho' (Sh), 'Akemi'. All cultivers could be classified as ZEP-1 type, ZEP-2 type and ZEP-1/-2 type. As an example, 'Seiho' is ZEP-1 type, 'Hirakisyu' and 'Clementine' are ZEP-2 type, and ZEP-1/-2 type is 'Miyagawa wase', 'Trovita' orange, and 'Kiyomi' tangor. (C) Segregation of fragment patterns in 'Kiyomi' (left), 'Miyagawa wase', and their progeny. Numbers in parentheses indicate segregation in the population analyzed. (D) Segregation of fragment patterns in 'Kiyomi' (left), 'Clementine', and their progeny. Numbers in parentheses indicate segregation in the population analyzed.

Reverse transcription-polymerase chain reaction (RT$P C R)$

Total RNA was extracted from juice sacs separated from fruits sampled at $30,60,120$, and 180 days after flowering (DAF) from 'Miyagawa wase' and 'Washington' navel orange (C. sinensis). Total RNA was extracted as described by Ikoma et al. (1996) and purified with an RNeasy Minikit (Qiagen) with on-column DNase digestion. First-strand cDNAs were synthesized from $5 \mu \mathrm{g}$ total RNA by reverse transcriptase with Oligo-(dT) primers using a Ready-To-Go You-Primed First Strand Kit (GE Healthcare). The ZEP-1/-2 sequence was used to design primers (Forward, 5'-AGATTTTGTTCCGGCT GATAT; Reverse, 5'-CCGAGTCCCCAAGCAA), not to 
Table 1. Primers used for direct sequencing and RT-PCR expression analyses, locus-specific TaqMan MGB probes, and allele-discriminating TaqMan probes/primers for Citrus ZEP genes.

\begin{tabular}{|c|c|c|c|}
\hline Purpose of primers & Intended locus/alleles & Primer and probe sequence ( $5^{\prime}$ to $3^{\prime}$ ) & Orientation \\
\hline \multirow{14}{*}{ Sequencing } & \multirow{6}{*}{$Z E P-1 /-2$ and $Z E P-3 /-4$} & TTTATTTGGGCCACAGGAAGC & Sense read \\
\hline & & TCCCCAAGCAAAGTAACACGA & Antisense read \\
\hline & & AAGGGCGAAGGTATAGGGTAT & Sense read \\
\hline & & AGAGCTAGGAGACTGCGAGTG & Sense read \\
\hline & & TTGGCTGCTAAAAGGAAAGGA & Sense read \\
\hline & & CCCTATACCTTCGCCCTTCAT & Antisense read \\
\hline & \multirow{6}{*}{$Z E P-1 /-2$} & AAGAGTAATTAGCCGAATG & Sense read \\
\hline & & GTTCCTTGTGAAATGCGTACC & Antisense read \\
\hline & & GCAGAGCGAACATGGAACCTA & Sense read \\
\hline & & GGAGTTCCGATCACCTTAACC & Antisense read \\
\hline & & GATACTTTCACTCCTGCAGCAGAA & Sense read \\
\hline & & CTTCGGGGTCATCCACTCCA & Antisense read \\
\hline & \multirow{2}{*}{$Z E P-3 /-4$} & TAAGTGTAGGACTTGGACCAC & Sense read \\
\hline & & GCTATTGGATGGACGAA & Antisense read \\
\hline \multirow{8}{*}{ Allele-specific RT-PCRs } & \multirow{4}{*}{$\begin{array}{l}Z E P-1 m /-2 m \text { of } \\
\text { 'Miyagawa wase' }\end{array}$} & TCGAGTGTTTCTAGGACACAAACAGT & Sense read \\
\hline & & TTTCAGCAACCTTTCCTTTTTACC & Antisense read \\
\hline & & AGTGGATG & TaqMan MGB probe (FAM probe) \\
\hline & & AGTGGATG $\underline{G C C C C G A}$ & TaqMan MGB probe (VIC probe) \\
\hline & \multirow{4}{*}{$\begin{array}{l}Z E P-1 o /-2 o \text { or }-2 m \text { of } \\
\text { 'Trovita' orange and } \\
\text { 'Kiyomi' tangor }\end{array}$} & GGGCTGGAAAAATGCAGTGGTA & Sense read \\
\hline & & GGCCATCCACTCCACCTG & Antisense read \\
\hline & & CTGGTTCCTTGTTAAAT & TaqMan MGB probe (FAM probe) \\
\hline & & CTGGTTCCTTGTGAAAT & TaqMan MGB probe (VIC probe) \\
\hline \multirow{3}{*}{$Z E P-1 /-2$ locus specific RT-PCR } & \multirow{3}{*}{$\begin{array}{l}Z E P-1 /-2 \text { of all } \\
\text { cultivars }\end{array}$} & TTCAGCGCAGGTGTCCAA & Sense read \\
\hline & & AATCAGGTAGAAGGCACCATCTTT & Antisense read \\
\hline & & ATGCATGCTCGTATCAG & TaqMan MGB probe \\
\hline
\end{tabular}

amplify $Z E P-3 /-4$; in contrast, the $Z E P-3 /-4$ sequence was used to design primers (Forward, 5'-TAAGTGTAGG ACTTGGACCAC; Reverse, 5'-GCTATTGGATGGAC GAA), not to amplify ZEP-1/-2, and RT-PCR was conducted using the AmpliTaq Gold (Applied Biosystems) protocol in a $20 \mu \mathrm{L}$ reaction volume for 25 PCR cycles. The PCR reaction mixtures were separated by electrophoresis on a $1.5 \%$ agarose gel and stained with EtBr solution. Amplified fragments were detected on a UV trans-illuminator.

Quantification of expression for allele-specific transcripts using TaqMan allele discrimination probel primer system

Total RNA was extracted from 'Miyagawa wase', 'Trovita' orange, and 'Kiyomi' tangor fruit harvested in November 2005, January 2006, and January 2008. Total RNA $(0.2 \mu \mathrm{g})$ was used to synthesize cDNAs with random hexamers at $37^{\circ} \mathrm{C}$ for $60 \mathrm{~min}$ using TaqMan reverse transcription reagents (Applied Biosystems).

First, the relative quantity of total $Z E P$ expression was evaluated using a TaqMan MGB probe with a set of primers/probe. We used Primer Express software (Applied Biosystems) to design primers from common sequences in ZEP cDNA among the three Citrus varieties. The primers were as follows; Forward, 5'-
TTCAGCGCAGGTGTCCAA; Reverse, 5'-AATCAGGT AGAAGGCACCATCTTT; and the MGB-Probe, 5'ATGCATGCTCGTATCAG. TaqMan Ribosomal RNA Control Reagents VIC Probe (Applied Biosystems) was used as an endogenous control. Each reaction contained $900 \mathrm{nM}$ primers, $250 \mathrm{nM}$ TaqMan MGB Probe, and $2.5 \mu \mathrm{L}$ template cDNA. The thermal cycling conditions were as follows: $95^{\circ} \mathrm{C}$ for $10 \mathrm{~min}$, followed by 40 cycles of $95^{\circ} \mathrm{C}$ for $15 \mathrm{~s}$ and $60^{\circ} \mathrm{C}$ for $60 \mathrm{~s}$. The levels of gene expression were analyzed with ABI PRISM 7300 Sequence Detection System Software (Applied Biosystems) and relative quantities of $Z E P$ expression were normalized against the expression of $18 \mathrm{~S}$ ribosomal RNA.

Next, Primer Express software (Applied Biosystems) was used to design TaqMan MGB probe sets labeled with VIC or FAM to discriminate single nucleotide polymorphisms (SNPs) among ZEP alleles in 'Miyagawa wase' and 'Trovita' orange. To evaluate the ratio of the expression of ZEP alleles, TaqMan allele discrimination PCR was carried out using the TaqMan Universal PCR Master Mix (Applied Biosystems) and an ABI PRISM 7300 (Applied Biosystems). Each reaction mixture contained $900 \mathrm{nM}$ primers, $200 \mathrm{nM}$ TaqMan FAM or VIC probes, and $2 \mu \mathrm{L}$ template cDNA. The thermal cycling conditions were the same as described for the total ZEP expression RT-PCR assay. The relative 
fluorescence levels of VIC and FAM were calibrated against the cDNA ratio. A calibration curve for each SNP detection probe was constructed as described by Katsumoto et al. (2005). For all alleles with an SNP site, partial ZEP cDNA sequences of the same length were cloned and purified. Two concentrations of cDNA, $10^{-5} \mathrm{ng} \cdot \mu \mathrm{L}^{-1}$ and $10^{-6} \mathrm{ng} \cdot \mu \mathrm{L}^{-1}$, were prepared for each clone. Then, homozygous samples were mixed to obtain mixtures with the following allele ratios: $10: 0,8: 2$, $6: 4,4: 6,2: 8$, and $0: 10$. Allele-specific expression ratios were normalized against each calibration curve. Real-time quantitative RT-PCR was performed for three replicates for each sample. Mean values $\pm \mathrm{SD}(n=3)$ are shown. For 'Kiyomi' tangor, probes and sets of primers were the same as those used for 'Trovita' orange.

\section{Results}

1. Analysis of genome sequences of ZEP genes in Citrus

1) Three types of ZEP gene sequences in 'Miyagawa wase'

A set of primers was designed from the conserved region of previously published $Z E P$ cDNA sequences (accession numbers AB114654 and AB075547). These primers produced three PCR fragments from 'Miyagawa wase' genomic DNA. The 887 bp, 1166 bp, and 1396 bp fragments were designated as ZEP-1, ZEP-2 and ZEP3 , respectively (Fig. 1A). As shown in Figure 1B, the ZEP-3 fragment was amplified from all cultivars, but ZEP-1 and ZEP-2 were not present in all citrus cultivars. Cultivars were classed as ZEP-1 type, ZEP-2 type, or $Z E P-1 /-2$ type on the basis of the presence or absence of ZEP-1 and ZEP-2. For example, ZEP-2 was present and ZEP-1 was absent in 'Clementine' (C. clementina) and 'Hirakisyu' ( $C$. kinokuni); hence, they were classed as ZEP-2 types. The ZEP-1 type 'Seiho' (a hybrid of 'Kiyomi' $\times$ 'Mineola') contained ZEP-1 but lacked ZEP2. To determine whether the fragments were derived from the same locus, we examined segregation in hybrid progenies derived from four different crosses. Progeny derived from 'Kiyomi' tangor (ZEP-1/-2) and 'Miyagawa wase' (ZEP-1/-2), segregated into three types: 4 individuals had ZEP-1 only; 6 had ZEP-2 only, and 11 had both ZEP-1 and ZEP-2 (Fig. 1C). Hybrid progeny derived from 'Kiyomi' tangor (ZEP-1/-2) and
'Clementine' (ZEP-2) segregated into ZEP-1/-2 and ZEP-2 types at $12: 9$ (Fig. 1D). Progeny derived from the other two crosses was also segregated into ZEP-1/2 and ZEP-2 or ZEP-1, ZEP-1/-2 and ZEP-2 groups (data not shown).

These segregation patterns suggest that ZEP-1 and ZEP-2 are amplification products derived from alleles at one locus (designated as $Z E P-1 /-2$ ) and that because ZEP-3 amplification products are detected in all progeny, ZEP-3 fragments are derived from another locus in the Citrus genome.

To confirm whether the PCR fragments were derived from $Z E P$ genes in the 'Miyagawa wase' genome, BAC clones containing the $Z E P$ gene were screened by PCR with the same primer pairs used for the 'Miyagawa wase' genomic PCR. Of the six PCR-positive clones, two contained ZEP-1 (BAC113-9G and BAC131-6G), one contained ZEP-2 (BAC150-3F), and two contained ZEP3 (BAC180-4G and BAC198-3A). In addition, one PCRpositive clone was obtained but the fragment size was approximately $2000 \mathrm{bp}$ (BAC212-6B); these clones were used for sequence analyses. To distinguish these genomic sequences from those of other Citrus cultivars, ZEP-1, ZEP-2, and ZEP-3 from 'Miyagawa wase' BAC clones were designated as ZEP-1m, ZEP-2m, and ZEP-3m, respectively.

The BAC clones were sequenced by primer extension from the $Z E P$ genomic region and read over $7995 \mathrm{bp}$ for ZEP-1m (AB548570) and $8274 \mathrm{bp}$ for $Z E P-2 m$ (AB548571), which fully covered the ZEP gene coding regions. The predicted coding sequences of $Z E P-1 \mathrm{~m}$ and $Z E P-2 m$ were identical to previously published cDNA sequences of Citrus (AB075547 and AB114654, respectively).

Like the Arabidopsis ZEP gene, the genomic sequences of $Z E P-1 m$ and $Z E P-2 m$ consisted of 16 exons and 15 introns (Fig. 2). The coding region of $Z E P-1 m$ had high sequence identity with that of $Z E P-2 m(>98 \%$ in deduced amino acids). The main difference between $Z E P-1 m$ and ZEP-2 $m$ was in the 10th exon, where a frameshift caused by an indel variation resulted in a different amino acid sequence in ZEP-1m (Fig. 3).

Sequence analyses showed a low level of nucleotide substitution among the coding sequences. We detected

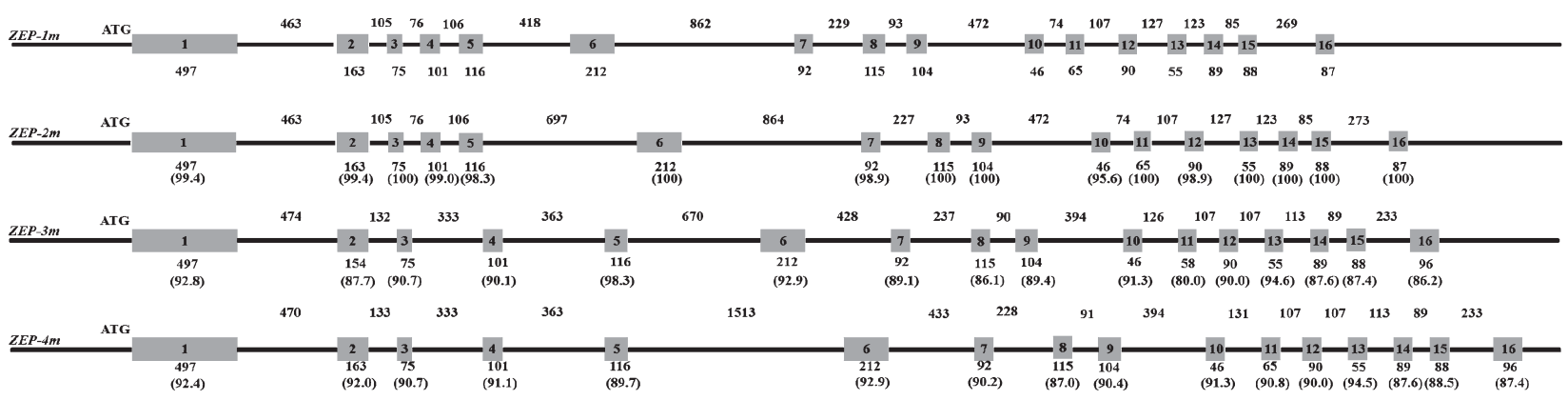

Fig. 2. Genome structure of $Z E P$ alleles of 'Miyagawa wase'. Boxes show exons predicted by cDNA sequence alignment. Numbers shown at or between boxes indicate lengths of exons/introns. Numbers in parentheses indicate percentage identity compared with $Z E P$ - $1 m$ sequence. 


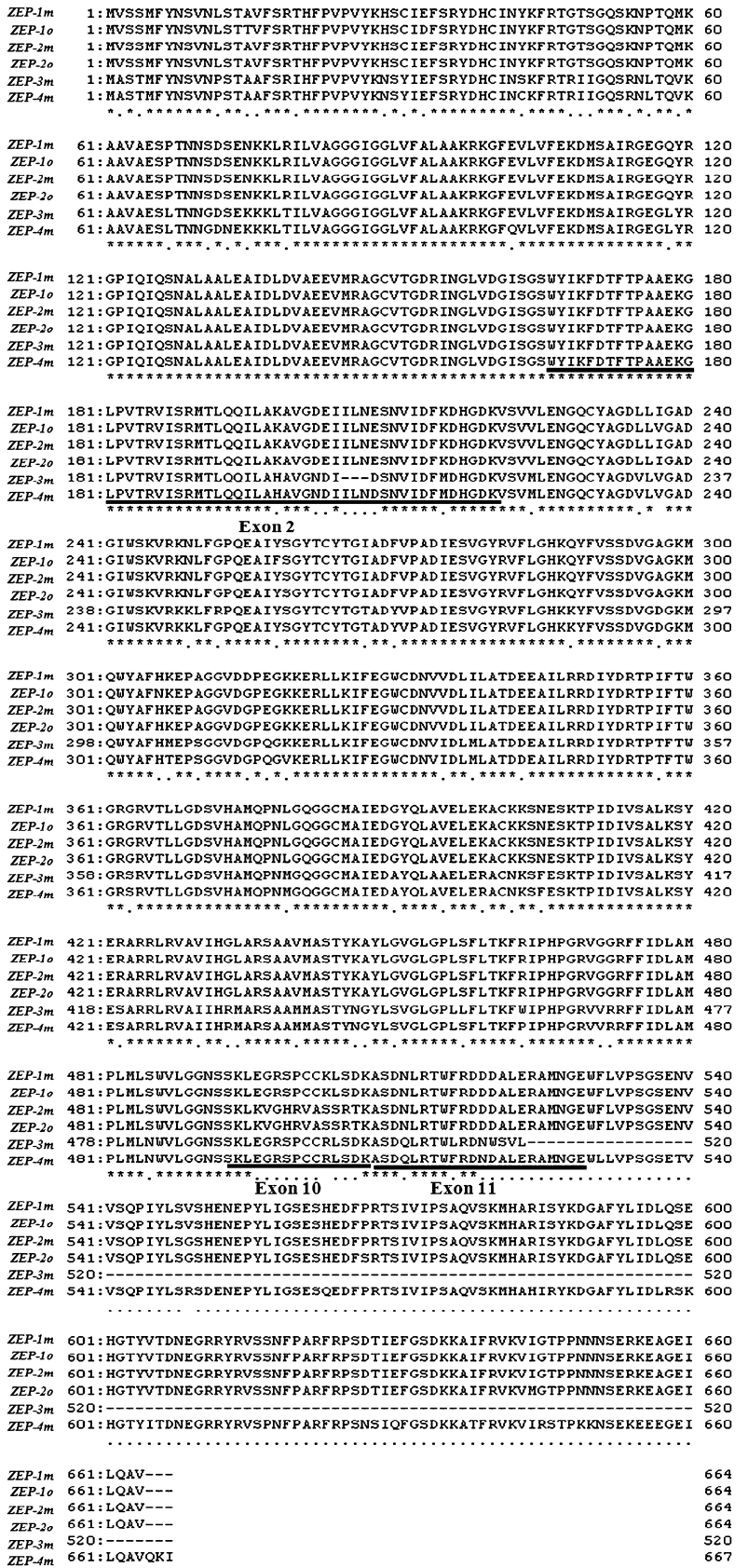

Fig. 3. Alignment of deduced amino acid sequence of $Z E P-1, Z E P$ 2, $Z E P-3 m$ and $Z E P-4 m$. These six sequences are $Z E P-1$ and $Z E P-2$ from 'Miyagawa wase' (suffix m) and 'Trovita' orange (suffix o), $Z E P-3 m$ and $Z E P-4 m$ from 'Miyagawa wase'. These six haplotypes showed high identity; however, in 10th exon, a frameshift caused by an indel variation resulted in a different amino acid sequence between $Z E P-1$ and $Z E P$-2. Although $Z E P$ $3 m$ and $Z E P-4 m$ sequences also showed high identities at CDSs, $Z E P-3 m$ had 9 bp and 7 bp deletion in 2th exon and 11th exon. This $7 \mathrm{bp}$ deletion at 11 th exon in ZEP-3m showed the appearance of a stop codon, which suggests a possible loss of function of $Z E P-3 m$.

36 SNPs in the $3609 \mathrm{bp}$ of intron sequences. The 5 th intron was 279 bp longer in ZEP-2m than in ZEP- $1 m$, corresponding to the differences in PCR fragment sizes. Compared with the transcribed region, there was much greater sequence variation in the $1736 \mathrm{bp} 5^{\prime}$ UTR sequences between $Z E P-1$ and $Z E P-2$ of 'Miyagawa wase'. Sequence identity between the 1736 bp 5' UTR sequences was $93.6 \%$ at the nucleotide level.

To confirm sequence diversity between $Z E P-1$ and $Z E P-2$ in other Citrus cultivars, genomic sequences for $Z E P-1$ and ZEP-2 were investigated in 'Trovita' orange, which was also heterozygous at the ZEP-1/-2 locus. Since then, $Z E P-1$ and $Z E P-2$, derived from 'Trovita' orange, have been described as ZEP-1o (AB548572) and $Z E P-2 o$ (AB548573). The PCR fragment length of ZEP$1 o$ from 'Trovita' orange was similar to that of ZEP- $1 \mathrm{~m}$. The entire gene was sequenced, including $1736 \mathrm{bp}$ of the 5' UTR and $669 \mathrm{bp}$ of the 3' UTR. Within the $8010 \mathrm{bp}$ region there were 4 indel sites, 20 SNP sites, and 2 base substitutions in the coding region as compared with $Z E P$ $1 m$. ZEP-2o from 'Trovita' orange was a typical ZEP2 type gene. An 8259 bp region was sequenced, and 8 indels and 21 SNPs were observed in the coding region, as compared with $Z E P-2 m$; however, as shown in Figure 3 , amino acid sequences were highly conserved within $Z E P-1$ types and $Z E P-2$ types. Genomic sequence analysis of $Z E P-1 /-2$ in a heterozygous cultivar 'Kiyomi' tangor (hybrid of 'Miyagawa wase' and 'Trovita' orange) clarified that 'Kiyomi' was inherited ZEP-1o from 'Trovita' orange, and $Z E P-2 m$ from 'Miyagawa wase'.

For $Z E P-3 m$, we sequenced an $7983 \mathrm{bp}$ region, including $5^{\prime}$ and $3^{\prime}$ UTRs on two BAC clones. The sequences of ZEP-3m (BAC180-4G and BAC198-3A) were very similar to $Z E P-1 /-2$; however, to date, no sequences corresponding to this type have been registered in public databases. To confirm whether the BAC clone (BAC212-6B) with $2239 \mathrm{bp}$ of PCR fragment has a $Z E P$-like sequence, the sequence covering $7744 \mathrm{bp}$ including 5 ' and $3^{\prime}$ UTRs was determined. The sequence was very similar to $Z E P-3 m$ and was therefore new sequence designated tentatively as $Z E P-4 m$ (Fig. 2).

To investigate the genomic structure of $Z E P-3 m$ and $Z E P-4 m$, a cDNA clone from young fruit (30 days after anthesis) of 'Miyagawa wase' was isolated and sequenced to confirm the coding regions. Sequence alignment of the cDNA sequence and $Z E P-3 m$ or ZEP$4 m$ showed that the coding sequences of $Z E P-3 m$ and $Z E P-4 m$ were divided into 16 exons, such as $Z E P-1 m$ and $Z E P-2 m$. The $Z E P-3 m$ genomic sequence differed from that of $Z E P-4 m$ mainly at the 2 th and 11 th exons. In $Z E P-3 m$, the 11 th exon contained a $7 \mathrm{bp}$ deletion, resulting in generation of a stop codon, which suggests the possible loss of function for $Z E P-3 m$ (Fig. 3). The predicted coding sequences of $Z E P-4 m$ showed $90.0 \%$ identity to $Z E P-1 m$ over the 16 exons, and $89.9 \%$ identity to $Z E P-2 m$ (Fig. 3). The 4th introns of $Z E P-3 m$ were longer than those of $Z E P-1 m$ and $Z E P-2 m$, corresponding to the longest PCR products amplified from Citrus genomic DNA (Fig. 1B).

Furthermore, the 5th intron of $Z E P-4 m$ was $843 \mathrm{bp}$ longer than that of $Z E P-3 m$, suggesting that because this 
insertion made it difficult to amplify the fragment of ZEP-4m, the longest genomic PCR fragment of ZEP$4 \mathrm{~m}$ was not detected (Fig. 1). ZEP-3m and ZEP-4m had very high sequence identity in the possible coding sequences, but whether $Z E P-3 m$ and $Z E P-4 m$ were in allellism has not been confirmed yet.

Cluster analysis using the $\mathrm{NJ}$ method showed that $Z E P-3 m /-4 m$ clustered more tightly with ZEP- $1 m$ and $Z E P-2 m$ of Citrus than with ZEPs from other plants. This suggested that $Z E P$ genes might have arisen from relatively recent duplication events in Citrus (Fig.4).

\section{Expression profiles of ZEP genes during fruit development in Citrus \\ 1) ZEP-1/-2 and ZEP-3/-4 expression in Citrus fruit}

The expression profiles of $Z E P$ genes in 'Miyagawa wase' and 'Washington navel' orange (C. sinensis) were evaluated by RT-PCR using specific primers that discriminated $Z E P-1 /-2$ from $Z E P-3 /-4$ but were common to $Z E P-1$ and $Z E P-2$ groups; in addition, the specific primers could not discriminate $Z E P-3 m$ from $Z E P-4 m$. As shown in Figure 5A, a low level of ZEP$4 m$ expression was detected in young 'Miyagawa wase' fruit (DAF30 and DAF60), but expression decreased below detection limits at DAF120 and at maturity (DAF180), while ZEP-3m, which has a stop codon at the 11th exon, was not detected at any stage of fruit development (data not shown). RT-PCR using specific primers that discriminate between ZEP-1/-2 and ZEP-3/

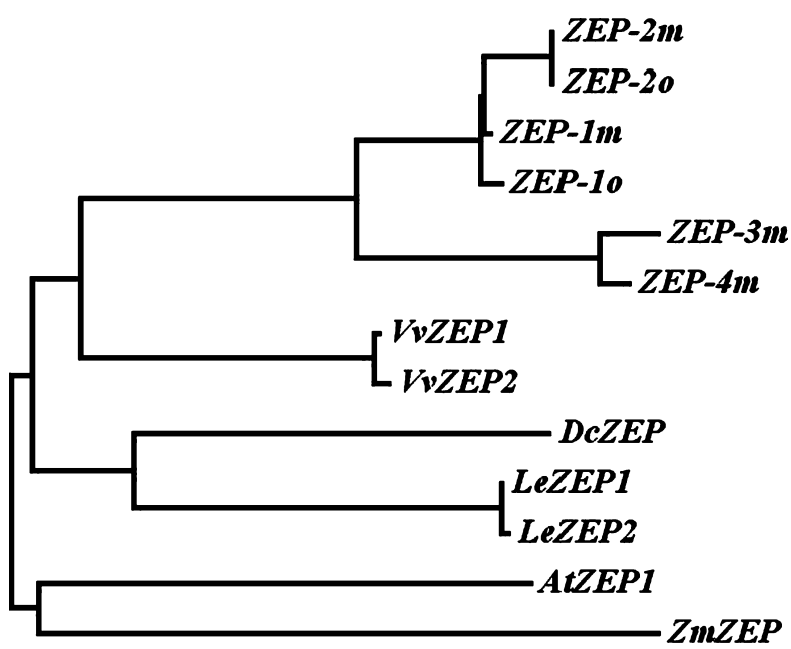

$$
\text { †.02 }
$$

Fig. 4. Phylogenic tree based on deduced amino acid sequences of ZEP genes constructed using MEGA4 Build\#: 4026 by the NJ method. Scale bar indicates distances (branch length) among compared sequences. Compared sequences: ZEP-1m (AB548570), ZEP-2m (AB548571), ZEP-3m, and ZEP-4m from C. unshiu, ZEP-1o (AB548572) and ZEP-2o (AB548573) from $C$. sinensis, and previously published sequences from $A$. thaliana (AtZEP, accession no. AF281655), grape (VvZEP1, XM_002282507; VvZEP2, AY337615), Zea mays (ZmZEP, EU970775), carrot (DcZEP, DQ192197), and tomato (LeZEP1, EF581828; LeZEP2, Z83835).
-4 was carried out to evaluate the expression of $Z E P-3 /$ -4 . The results showed that only $Z E P-4 m$ was expressed.

The expression patterns of $Z E P-1 \mathrm{~m} /-2 \mathrm{~m}$ differed from that of $Z E P-4 m$ (Fig. 5B). Expressions of both $Z E P-1 m$ and $Z E P-2 m$ increased from immature (DAF60) to mature fruit (DAF180) in 'Miyagawa wase' fruit, but peaked level in DAF180 fruit. The expression profiles of ZEP-1/-2 and ZEP-3/-4 in 'Washington navel' fruit were similar to those in 'Miyagawa wase' (data not shown). These results suggest that $Z E P-1 /-2$ have important roles in determining the $Z E P$ expression level in mature Citrus fruit, but $Z E P-3 /-4$ does not.

Next, total transcript levels of $Z E P-1 /-2$ were estimated quantitatively by RT-PCR using a TaqMan probe that simultaneously detected the expression of $Z E P-1$ and ZEP-2 in maturing fruit (November 2005 to January 2006). Levels of $Z E P-1 /-2$ transcripts decreased from November (DAF180) to January (DAF240) in 'Miyagawa wase' and 'Trovita' orange fruit. The decrease was more rapid in 'Miyagawa wase', in which the transcript level in January was $62.7 \%$ of that in November. In 'Trovita' orange, the transcript level in January was $88.2 \%$ of that in November; therefore, the transcript level in 'Miyagawa wase' was lower than that in 'Trovita' orange in January, although that in 'Miyagawa wase' was higher than that in 'Trovita'

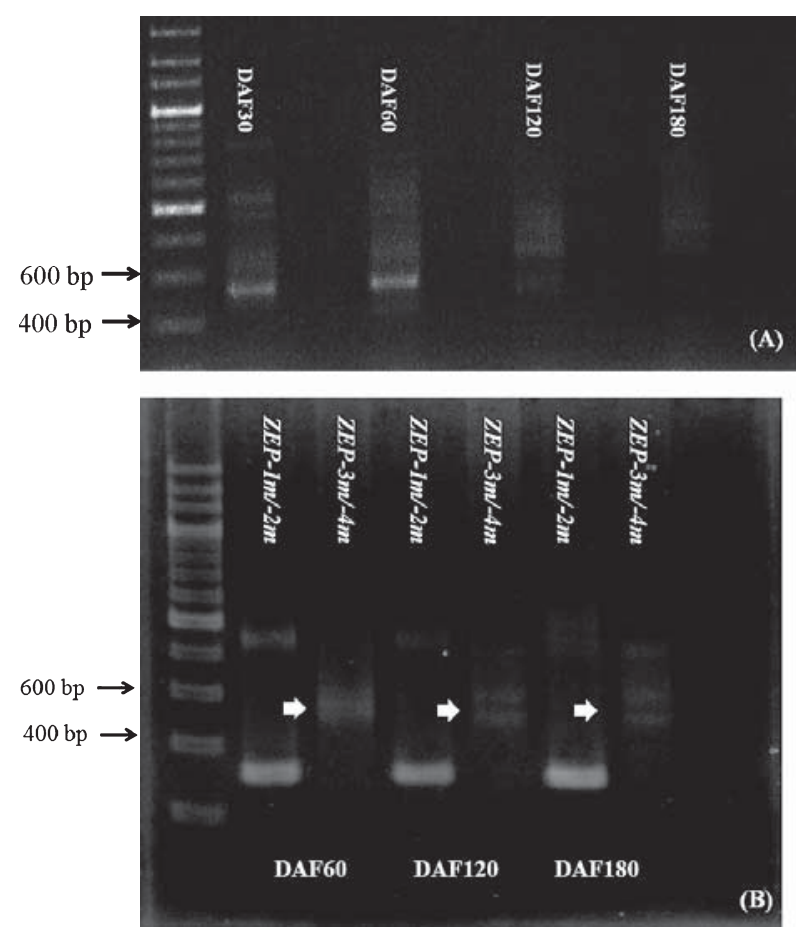

Fig. 5. RT-PCR analysis of $Z E P-4 m$ expression during fruit development in 'Miyagawa wase'. (A) $Z E P-4 m$ expression during fruit development. $Z E P-4 m$ was expressed in immature fruit and, as fruit matured, $Z E P-4 m$ expression progressively weakened. (B) Comparison of $Z E P-1 m /-2 m$ and $Z E P-4 m$ expression intensity. This showed that the expression levels of $Z E P-4 m$ were much weaker than $Z E P-1 m /-2 m$. This result was common during fruit development. 
orange in November (Fig. 6).

2) Allelic differences of ZEP-1/-2 expression in maturestage fruit

To determine the transcript ratio of ZEP-1:ZEP-2 at fruit maturity, we estimated expression levels in the fruit of three heterozygous cultivars, 'Miyagawa wase' (ZEP$1 m / Z E P-2 m)$, 'Trovita' orange $(Z E P-1 o / Z E P-2 o)$, and their hybrid 'Kiyomi' (ZEP-1o/ZEP-2m) using SNP discrimination. The TaqMan probe set was allelespecific, and detected both ZEP-1 and ZEP-2. As shown in Table 2, expression levels of the two alleles differed among the three cultivars in maturing fruit collected in November 2005, January 2006, and January 2008. In 'Miyagawa wase' fruit harvested in November 2005, $Z E P-1 m$ accounted for $59 \%$ of total ZEP-1/-2 gene expression, while $Z E P-2 m$ accounted for the remaining $41 \%$. The $Z E P-1 /-2$ ratio was very similar in January 2006 (ZEP-1m: 60\%, ZEP-2m: 40\%; Table 2). In 'Trovita' orange fruit, ZEP-1o was expressed at higher levels than ZEP-2o (69 and 31\%, respectively, in November 2005; and 72 and $28 \%$, respectively, in January 2006). The heterozygous 'Kiyomi' tangor containing both $Z E P-10$ and $Z E P-2 m$ showed large differences in expression levels between the alleles. In

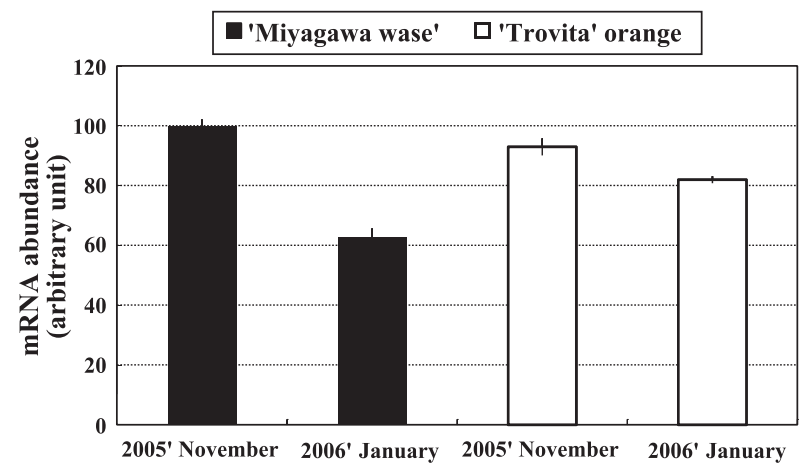

Fig. 6. Total expression of $Z E P-1$ and $Z E P-2$ in 'Miyagawa wase' and 'Trovita' orange at 2 months. Levels of allele-specific expression were analyzed by TaqMan real-time quantitative RTPCR. Probes and sets of primers used for analyses are shown in Table 1. Horizontal bars indicate SD $(\mathrm{n}=3)$. In these two varieties, the expressions of ZEP-1 and ZEP-2 declined in January compared with in November, while, the decline in expression in 'Miyagawa wase' was greater than in 'Trovita' orange.
'Kiyomi' fruit harvested in November 2005, ZEP-1o derived from 'Trovita' orange accounted for $75.5 \%$ of total expression and $Z E P-2 m$ from 'Miyagawa wase' accounted for the remaining $24.5 \%$. A similar expression rate was observed in fruit harvested in January, 2006 (ZEP-1o: 76.6\%, ZEP-2m: 23.4\%, Table 2). Differences in expression levels between alleles were confirmed in fruit harvested in 2008 (67:33 ZEP-1o:ZEP-2o expression ratio in 'Trovita' orange fruit; $74: 26 Z E P$ $1 o: Z E P-2 m$ expression ratio in 'Kiyomi' tangor fruit). Therefore, the expression ratio of $Z E P-1 /-2$ alleles in Citrus cultivars was maintained at similar levels in different fruiting seasons, although the total transcript levels of ZEP-1/-2 changed as fruit matured.

\section{Sequence diversity of 5' untranslated regions (UTRs) at ZEP-1/-2}

To examine structural differences in cis-element regions in relation to expression levels of ZEP genes, two $1783 \mathrm{bp}$ genomic sequences located in the 5 ' UTR of ZEP-1/-2 genes from 'Trovita' orange were compared with those from 'Miyagawa wase' BAC sequences. Within this region, the lowest identity was between $Z E P$ $1 m$ and ZEP-2o (93.5\%). The four haplotype sequences had relatively high identity in this region, but there were many SNPs, 1-30 bp indels, and replacements at two or more nucleotides. The sequence-level variations among the four haplotypes resulted in different motif structures of possible cis-regions.

Using PLACE (http://www.dna.affrc.go.jp/PLACE/ index.html), the possible motifs were surveyed for the four haplotype sequences and annotated as shown in Figure 7. We observed (TAA) and (TAATGA) repeats in the region from -489 to $-540 \mathrm{bp}$ from ATG in ZEP- $1 \mathrm{~m}$. These repeated motif sequences are known as POLASIG3, IBOXCORE and GATABOX, respectively (Fig. 7). The repeat structure of the two motifs in the various alleles was as follows: $(\mathrm{TAA})_{5}(\mathrm{TAATGA})_{4}$ for $Z E P-1 m$; (TAA) $)_{6}(\text { TAATGA })_{1}$ for $Z E P-2 m ;(\text { TAA })_{7}$ $(\text { TAATGA })_{5}$ for $Z E P-1 o$; and (TAA) $)_{7}(\text { TAATGA) })_{1}$ for $Z E P-2 o$. Compared with ZEP-1o, the sequence of this repeat domain was $12 \mathrm{bp}$ shorter in $Z E P-1 \mathrm{~m}, 24 \mathrm{bp}$ shorter in $Z E P-2 m$, and $30 \mathrm{bp}$ shorter in ZEP-2o.

There were many variations in the presence or absence of motif sequences among the four haplotypes. Almost

Table 2. Allele-specific expression ratio (expressed as percentage) of ZEP- 1 and $Z E P-2$ in three Citrus varieties. Levels of allele-specific expression were analyzed by TaqMan real-time quantitative RT-PCR. Probes and sets of primers used for analyses are shown in Table 1. Data are means $\pm \mathrm{SD}(\mathrm{n}=3)$.

\begin{tabular}{|c|c|c|c|c|c|c|}
\hline & \multicolumn{2}{|c|}{ 'Miyagawa wase' } & \multicolumn{2}{|c|}{ 'Trovita' orange } & \multicolumn{2}{|c|}{ 'Kiyomi' } \\
\hline & $Z E P-1 m$ & $Z E P-2 m$ & ZEP-1o & $Z E P-20$ & $Z E P-1 o$ & $Z E P-2 m$ \\
\hline 2005 November & 59.1 & 40.9 & 69.1 & 30.9 & 75.5 & 24.5 \\
\hline 2006 January & 60.3 & 39.7 & 72.0 & 28.0 & 76.6 & 23.4 \\
\hline 2008 January & - & - & 67.4 & 32.6 & 74.4 & 25.6 \\
\hline Average & 59.7 & 40.3 & 69.5 & 30.5 & 75.5 & 24.5 \\
\hline SD & 0.6 & 0.6 & 1.3 & 0.9 & 0.6 & 0.6 \\
\hline
\end{tabular}




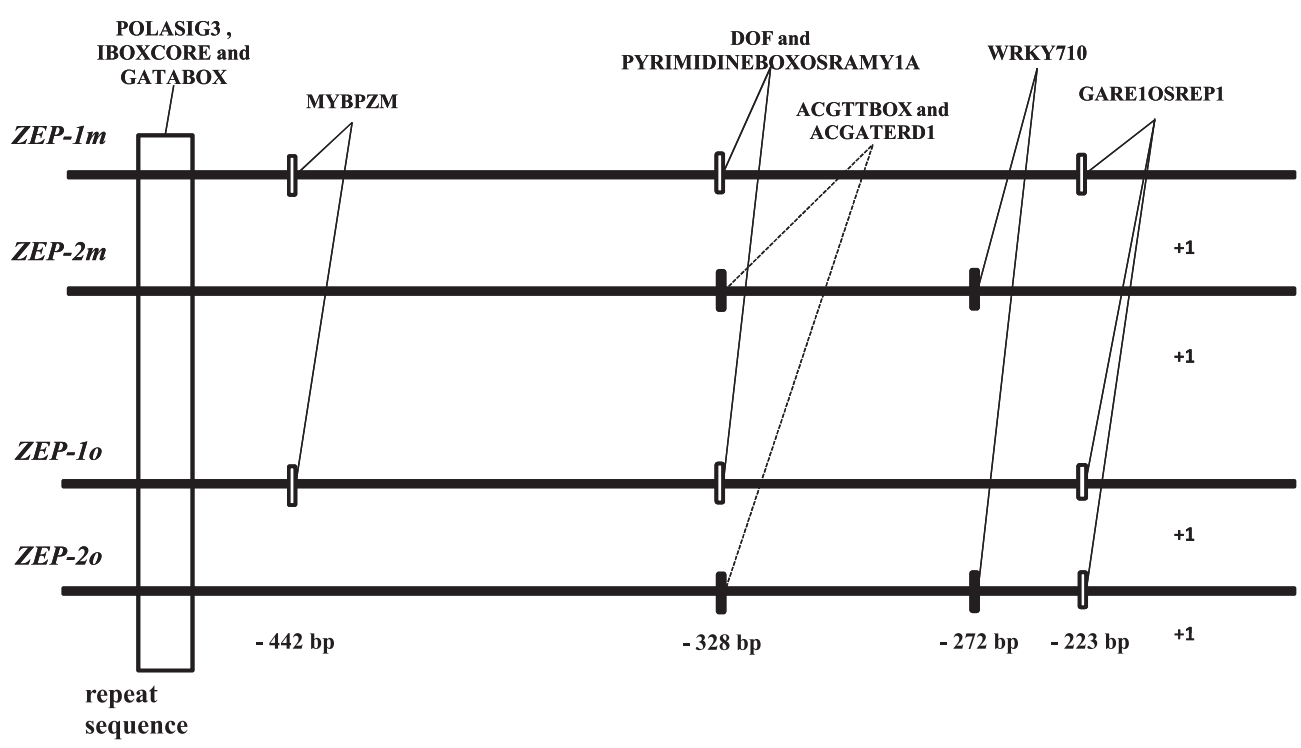

Fig. 7. Diagram of upstream regions of $Z E P$ genes ( $-600 \mathrm{bp}$ to +1 from translation start site). Nucleotide position shown relative to the translation start site. GARE1OSREP1 had three haplotypes other than ZEP-2m. This motif is "Gibberellin-responsive element (GARE)" found in the promoter region of a cystein proteinase (REP-1) gene in rice. PYRIMIDINEBOX found in rice alpha-amylase gene. ACGTTBOX is an ACGT element. ACGATERD1 is ACGT sequence (from -155 to -152) required for etiolation-induced expression of erd1 (early responsive to dehydration) in Arabidopsis. MYBPZM motif is the core region of the consensus maize P binding site. Maize P gene encodes red pigmentation of kernel pericarp, cob, and other floral organs. Number of (TAA) (TAATGA) $_{\mathrm{y}}$ repeats differed among the four haplotypes in the repeat sequence region, and, POLASIG3, IBOXCORE and GATABOX motifs were confirmed. The number of these three motifs in the repeat sequence region differed among haplotypes. Sequences were registered in the DDBJ: ZEP-1m, AB548570; ZEP-2m, AB548571; ZEP-1o, AB548572; ZEP-2o, AB548573.

all variations were haplotype-specific, but not alleletype-specific; however, some features were restricted to either ZEP-1 type or ZEP-2 type alleles. In ZEP- $1 \mathrm{~m}$ and ZEP-1o, MYB, DOF and PYRIMIDINEBOXOSRAMY1A motifs were commonly detected around $-500 \mathrm{bp}$ from the ATG codon, while $Z E P-2 m$ and $Z E P-2 o$ alleles lacked these motifs. On the other hand, ZEP-2 alleles had ACGTTBOX and ACGTATERD1 motifs (Fig. 7). Furthermore, ZEP-1 alleles had an MYBPZM motif, which is the core of a consensus maize $\mathrm{P}$ (Myb homolog) binding site, at -442 bp, while ZEP-2 alleles did not. In contrast, ZEP2 type alleles had a WRKY710 motif located at $-272 \mathrm{bp}$.

\section{Discussion}

$Z E P$ is a carotenoid biosynthetic gene encoding an enzyme with crucial roles in determining the level and composition of carotenoids. In grape and tomato, there are two $Z E P$ sequences in public databases but they did not seem to be paralogs because they showed very high identities with each other, with more than $99 \%$ identity at the amino acid level. In Citrus fruit, Kato et al. (2004) showed that the expression of ZEP increased during fruit maturation in Satsuma mandarin and 'Valencia' orange, and that expression levels differed between the two clutivars; however, not only cultivar-specific or genotype-specific mechanisms controlling ZEP expression, but also, from our results, the presence of a possible paralogous $Z E P$ gene with sequence diversity in plants, remain unclear.
Here, we analyzed in detail the allelic structure and expression of ZEP genes in Citrus. Our results provide new information about the control of ZEP gene expression.

\section{Newly identified ZEP gene in Citrus}

In Arabidopsis, two genomic DNA sequences with 99.7\% identity were identified from the DDBJ database (AF134577 and AF283761). To date, only one ZEP gene located at one locus on chromosome 5 (At5g67030) has been identified in Arabidopsis, and no other ZEP gene homologs have been reported.

In this study, four different genomic sequences were isolated from the BAC library of 'Miyagawa wase' (C. unshiu) and sequenced. Two of these sequences $(Z E P-1 m$ and $Z E P-2 m)$ were similar to cDNA sequences registered in the DDBJ database (AB114654 and $\mathrm{AB}$ 075547), and were very similar; however, the other two sequences showed a conserved exon/intron structure of the ZEP gene, but had longer 4th introns than other ZEP genes. Their predicted CDSs had $90 \%$ nucleotide identity with previously published cDNA sequences.

Progeny tests and comparisons of PCR fragment size from the 4th to 6th exon among cultivars suggested that the former two genomic sequences were alleles at the $Z E P-1 /-2$ locus, which had identical coding sequences with previously registered cDNA in the DDBJ. On the other hand, whether ZEP-3/-4 were in allelism in the second locus of $Z E P$ has not been confirmed although they had very similar coding sequences. Phylogenetic 
analyses of four $Z E P$ sequences in Citrus suggested that $Z E P-3 /-4$ were separated from $Z E P-1 /-2$ before ZEP-1 and $Z E P-2$ speciated; however, both $Z E P-3 /-4$ and $Z E P$ $1 /-2$ sequences in Citrus were clustered more tightly than ZEP sequences from other plants. Since both Satsuma mandarin and sweet orange were heterozygous in the $Z E P-1 /-2$ locus, $Z E P-3 /-4$ should be located in other loci. While $Z E P-1 /-2$ were well characterized by sequencing and expression analysis in Citrus (Kato et al., 2004), there is information on ZEP-3/-4 in Citrus. ZEP-4 was weakly expressed only at early stages of fruit development (DAF30 to DAF120) in contrast with ZEP1/-2 expression. Compared with ZEP-1/-2,ZEP-3/-4 may contribute differently to carotenoid metabolism in Citrus, but it may have a minor contribution to violaxanthin accumulation in fruit. Komatsu et al. (1999) reported the differential expression of three sucrose-phosphate synthase (SPS) isoforms during sucrose accumulation in developing citrus fruit. Their result was that one of three genes showed a differential expression pattern compared with the other two SPS genes; in addition, the transcription levels of the two SPS genes paralleled the activity of SPS. It would be interesting to know whether the two classes of ZEP genes with different expression profiles were generated similarly to the SPS isoforms.

These results suggest that $Z E P-4$ is an isoform with a different expression pattern from those of $Z E P-1 /-2$ as well as sequence similarity. How ZEP-3/-4 were generated in Citrus should be clarified in the future in addition to the contribution to carotenoid metabolism in whole plant organs, such as leaf, stem or root.

\section{Uneven transcript levels of ZEP-1/-2 alleles}

As reported previously (Kato et al., 2004), the expression of ZEP varied throughout fruit development and from year to year. In this study, we observed that ZEP expression in 'Miyagawa wase' fruit was higher than in 'Trovita' orange from November to January, which was not consistent with results reported previously by Kato et al. (2004); however, the tendency for a decreased expression level toward the maturity of ZEP in all cultivars was consistent with their results. In addition to the variation among cultivars in the expression of ZEP during fruit development and maturation, the results detected that allelic combination is one of the factors affecting variable $Z E P$ expression. The results showed that $Z E P-1$ alleles were expressed at 1.5- to 3-fold higher levels than ZEP-2 alleles in all three heterozygous cultivars examined. The expression ratios were fairly constant as fruit matured; and in different fruit growing seasons. To date, there have been no reports on the differential expression of $Z E P-1 /-2$ gene alleles in Citrus. There are some reports on different expression patterns between alleles in the other genes of plants. In C. unshiu, alleles of a heterozygous UDPglucose, limonoid glucosyl transferase gene, were expressed differently (Kita et al., 2003). In $\mathrm{F}_{1}$ hybrids of poplar, 17 out of 30 analyzed genes showed 1.5 -fold differences between expressions of the two alleles (Yan and Adams, 2007). Similarly, 43-53\% genes showed unequal allelic expression in maize (Springer and Stupar, 2007a, 2007b); however, the mechanisms that regulate the differential expression of the two alleles remain unclear.

The genotype of the Citrus hybrid cultivar 'Kiyomi' was a combination of ZEP-1o, the stronger allele from 'Trovita' orange, and ZEP-2m, the weaker allele from 'Miyagawa wase'. Compared with either of its parents, 'Kiyomi' showed much larger differences between the expression levels of the two alleles. This result suggests that the differences between the expression levels of the two alleles were caused at least partly by differences in cis-structures located in the 5' UTR of ZEP genes, as indicated below.

\section{Comparison of $5^{\prime}$ untranslated regions of ZEP genes}

For carotenoid metabolism genes, RAP2.2, a member of the AP2/ethylene-responsive element-binding protein gene, was only reported to act on the promoter region of PSY and PDS (Welsch et al., 2008). The report suggested that diversity among cis-elements as well as transcription factor genes would contribute to the genotype-dependent expression of genes involved in carotenogenesis; however, there has been no such analysis in the ZEP gene in Citrus.

The 5' UTRs of ZEP genes from 'Miyagawa wase' and 'Trovita' orange were compared to associate the allelic sequence differences to the expression in heterozygous plants. ZEP-1 alleles were transcribed at higher levels than $Z E P-2$ alleles; therefore, we selected sequence motifs that were common within $Z E P$ - 1 s but differed from $Z E P-2 \mathrm{~s}$. As a ZEP-1-specific motif, we detected motifs related to Myb-binding sites, including the Myb-related MYBPZM motif in ZEP-1 type haplotypes at $-442 \mathrm{bp}$. The MYBPZM motif was absent from $Z E P-2$ type alleles. A Myb-transcriptional factor is involved in regulating the gene expression in grape (Kobayashi et al., 2004) and apple (Ban et al., 2007) during anthocyanin biosynthesis, but it is still unknown whether Myb-like factors have roles in regulating the expression of ZEP or carotenoid biosynthesis genes, and how the sequence variations in Myb-sites in 5' UTRs are correlated with expression levels among alleles of the Citrus ZEP gene; however, the sequence structure of the possible $c i s$-region and its variation would provide important information to investigate the regulatory mechanism in the near future.

In addition to the potential of molecular analysis to improve the regulation of $Z E P$ gene expression, sequence diversity would be utilized to generate molecular markers to manipulate total ZEP expression and carotenoid components for breeding. Hybrids having weak allele combination might be selected by identifying the presence or absence of candidate elements, such as Myb 
sites, to accumulate zeaxanthin or $\beta$-cryptoxanthin in fruit. The functional site was discovered on a $M y b$ promoter region to regulate fruit rind color in grape (Kobayashi et al., 2004) and on an ACC synthase gene promoter to discriminate ethylene production in apple and Japanese pear fruit (Harada et al., 1997; Itai et al., 1999). However, beside cis-regulation, it is thought that various factors are involved in the gene expression, and the existence of trans-regulation or regulation factors of mRNA cannot be disregarded. This approach only clarified part of the mechanism of gene expression, and although the expression levels of ZEP are regulated in a quantitative manner in Citrus, further statistical analyses should be performed to confirm the effect of the functional sites in the cis-region on carotenoid metabolism, and other regulation factors should be investigated; that is, cis-element, transcription factors, regulation factor of mRNA and so on. When considering differences in expression levels, various factors should be considered comprehensively. From this result, because haplotypes are thought to be factors affecting gene expressions, they should be considered at breeding selection.

\section{Literature Cited}

Ban, Y., C. Honda, Y. Hatsuyama, M. Igarashi, H. Bessho and T. Moriguchi. 2007. Isolation and functional analysis of a MYB transcription factor gene that is a key regulator for the development of red coloration in apple skin. Plant Cell Physiol. 48: 958-970.

Bouvier, F., R. A. Backhaus and B. Camara. 1998. Induction and control of chromoplast-specific carotenoid genes by oxidative stress. J. Biol. Chem. 273: 30651-30659.

Bouvier, F., A. d'Harlingue, P. Hugueney, E. Marin, A. MarionPoll and B. Camara. 1996. Xanthophyll biosynthesis. Cloning, expression, functional reconstitution, and regulation of betacyclohexenyl carotenoid epoxidase from pepper (Capsicum annuит). J. Biol. Chem. 271: 28861-28867.

Busch, M., A. Seuter and R. Hain. 2002. Functional analysis of the early steps of carotenoid biosynthesis in tobacco. Plant Physiol. 128: 439-453.

Fraser, P. D., M. R. Truesdale, C. R. Bird, W. Schuch and P. M. Bramley. 1994. Carotenoid biosynthesis during tomato fruit development: Evidence for tissue-specific gene expression. Plant Physiol. 105: 405-413.

Giuliano, G., G. E. Bartley and P. A. Scolnik. 1993. Regulation of carotenoid biosynthesis during tomato development. Plant Cell 5: 379-387.

Goodner, K. L., R. L. Rouseff and H. J. Hofsommer. 2001. Orange, mandarin, and hybrid classification using multivariate statistics based on carotenoid profiles. J. Agric. Food Chem. 49: $1146-1150$.

Gross, J. 1987. Carotenoid: pigment in fruit. Academic Press, London.

Harada, T., T. Sunako, W. Sakuraba, S. Goto, M. Senda, S. Akada and M. Niizeki. 1997. Genomic nucleotide sequence of a ripening-related 1-aminocyclopropane-1-carboxylate synthase gene (MdACS-1) in apple. Plant Physiol. 113: 1465.

Ikoma, Y., A. Komatsu, M. Kita, K. Ogawa, M. Omura, M. Yano and T. Moriguchi. 2001. Expression of a phytoene synthase gene and characteristic carotenoid accumulation during citrus fruit development. Physiol. Plant. 111: 232-238.

Ikoma, Y., M. Yano, K. Ogawa, T. Yoshioka, Z. C. Xu, S. Hisada, M. Omura and T. Moriguchi. 1996. Isolation and evaluation of RNA from polysaccharide-rich tissues in fruit for quality by cDNA library construction and RT-PCR. J. Japan. Soc. Hort. Sci. 64: 809-814.

Isaacson, T., G. Ronen, D. Zamir and J. Hirschberg. 2002. Cloning of tangerine from tomato reveals a carotenoid isomerase essential for the production of $\beta$-carotene and xanthophylls in plants. Plant Cell 14: 333-342.

Itai, A., T. Kawata, K. Tanabe, F. Tamura, M. Uchiyama, M. Tomomitsu and N. Shiraiwa. 1999. Identification of 1aminocyclopropane-1-carboxylic acid synthase genes controlling the ethylene level of ripening fruit in Japanese pear (Pyrus pyrifolia Nakai). Mol. General Genet. 262: 42-49.

Kato, M., Y. Ikoma, H. Matsumoto, M. Sugiura, H. Hyodo and M. Yano. 2004. Accumulation of carotenoids and expression of carotenoid biosynthetic genes during maturation in citrus fruit. Plant Physiol. 134: 824-837.

Katsumoto, H., M. Ito, O. Sakatsume and I. Ieiri. 2005. Allelespecific transcript quantification using $\operatorname{TaqMan}^{\circledR}$ PCR method. The 28th Annual Meeting of the Molecular Biology Society of Japan 3P-1142 (In Japanese).

Kita, M., T. Endo, T. Shimada, T. Moriguchi, Y. Hirata, S. Hasegawa and M. Omura. 2003. Allelic structure of UDPglucose: limonoid glucosyltransferase affect limonoid bitterness in Citrus unshiu and C. sinensis. Euphytica 132: 87-94.

Kim, I. J., K. C. Ko, C. S. Kim and W. I. Chung. 2001. Isolation and characterization of cDNAs encoding $\beta$-carotene hydroxylase in Citrus. Plant Sci. 161: 1005-1010.

Kobayashi, S., N. Goto-Yamamoto and H. Hirochika. 2004. Retrotransposon-induced mutations in grape skin color. Science 304: 982.

Komatsu, A., Y. Takanokura, T. Moriguchi, M. Omura and T. Akihama. 1999. Differential expression of three sucrosephosphate synthase isoforms during sucrose accumulation in citrus fruits (Citrus unshiu Marc.). Plant Sci. 140: 169-178.

Lee, H. S. and W. S. Castle. 2001. Seasonal changes of carotenoid pigments and color in Hamlin, Earlygold, and Budd Blood orange juices. J. Agric. Food Chem. 49: 877-882.

Molnár, P. and J. Szabolcs. 1980. $\beta$-Citraurin epoxide, a new carotenoid from Valencia orange peel. Phytochemistry 19: 633-637.

Nishino, H., H. Tokuda, M. Murakoshi, Y. Satomi, M. Masuda, M. Onozuka, S. Yamaguchi, J. Takayasu, J. Tsuruta, M. Okuda, F. Khachik, T. Narisawa, N. Takasuka and M. Yano. 2000. Cancer prevention by natural carotenoids. Biofactors 2000 13: 89-94.

Olson, J, A. 1989. Provitamin-A function of carotenoids: the conversion of $\beta$-carotene into vitamin-A. J. Nutr. 119: 105 108.

Omura, M., T. Ueda, M. Kita, A. Komatsu, Y. Takanokura, T. Shimada, T. Endo-Inagaki, H. Nesumi and T. Yoshida. 2000. EST mapping of Citrus. Proc. Intl. Soc. Citriculture for IX Congr. 2000: 71-74.

Park, H., S. Kreunen, A. J. Cuttriss, D. DellaPenna and B. J. Pogson. 2002. Identification of the carotenoid isomerase provides insight into carotenoid biosynthesis, prolamellar body formation, and photomorphogenesis. Plant Cell 14: 321332.

Pogson, B., K. A. McDonald, M. Truong, G. Britton and D. DellaPenna. 1996. Arabidopsis carotenoid mutants demonstrate that lutein is not essential for photosynthesis in higher plants. Plant Cell 8: 1627-1639. 
Ronen, G., M. Cohen, D. Zamir and J. Hirschberg. 1999. Regulation of carotenoid biosynthesis during tomato fruit development: expression of the gene for lycopene epsilon-cyclase is downregulated during ripening and is elevated in the mutant Delta. Plant J. 17: 341-351.

Shimada, T., F. Nishikawa, T. Endo, H. Fujii, Y. Nobata, T. Shimizu and M. Omura. 2005. Construction and initial evaluation of BAC library for Citrus unshiu Marc. 'Miyagawa wase'. Abstr. Japan. Soc. Hort. Sci. Spring Meet. 74: 187 (In Japanese).

Springer, N. M. and R. M. Stupar. 2007a. Allele-specific expression patterns reveal biases and embryo-specific parent-of-origin effects in hybrid maize. Plant Cell 19: 2391-2402.

Springer, N. M. and R. M. Stupar. 2007b. Allelic variation and heterosis in maize: how do two halves make more than a whole? Genome Res. 17: 264-275.

Welsch, R., J. Medina, G. Giuliano, P. Beyer and J. von Lintig. 2003. Structural and functional characterization of the phytoene synthase promoter from Arabidopsis thaliana. Planta 216: 523-534.

Welsch, R., D. Maass, T. Voegel, D. Dellapenna and P. Beyer. 2008. Transcription factor RAP2.2 and its interacting partner SINAT2: stable elements in the carotenogenesis of Arabidopsis leaves. Plant Physiol. 145: 1073-1085.

Yan, Z. and K. L. Adams. 2007. Extensive allelic variation in gene expression in populus $\mathrm{F}_{1}$ hybrids. Genetics 177: 1987-1996. 